\title{
Resonance of Transverse Capillary and Longitudinal Waves as a Tool for Monolayer Investigations at the Air/Water Interface
}

\author{
Viola Vogel* and Dietmar Möbius \\ Max-Planck-Institut für biophysikalische Chemie, Am Fassberg, 3400 Göttingen, FRG \\ Received February 12, 1988. In Final Form: September 16, 1988
}

\begin{abstract}
Periodical surface density fluctuations, i.e., longitudinal waves, and transverse capillary waves are inherently coupled at film-covered liquid-gas interfaces. A resonance occurs for a particular surface dilatational elasticity where both waves have the same propagation velocity. In this paper, periodical surface density fluctuations are excited in polymer monolayers via the generation of transverse capillary waves. Without contacting the monolayer these transverse ripples are excited by an electrocapillary deflection of the interface $(100$ $\mathrm{Hz}$ to $1 \mathrm{kHz}$ ) and detected by specular reflection of a laser beam from the water surface. The surface pressure fluctuations are investigated by superposing two transverse capillary waves perpendicularly to each other under resonance. In addition, the surface pressure/area isotherm as well as the propagation characteristics of transverse capillary waves are given for a poly(vinyl acetate) monolayer.
\end{abstract}

\section{Introduction}

Studies on the propagation of surface density fluctuations in lipid monolayers at the water/air interface may provide a basis for the investigation of relaxation processes as well as signal transduction in monolayers and membranes.

Laterally oscillating bars have been used to compress and expand monolayers periodically, i.e., to excite periodical longitudinal waves. ${ }^{1-3}$ Oscillating bars always have the disadvantage not only to disrupt the monlayer but also to penetrate into the incompressible subphase. Further, a common technique for the observation of surface density fluctuations has been to follow the lateral displacement of small particles sprinkled on top of the monolayer. ${ }^{2,4}$ They may contaminate the interface and disturb the wave propagation since they are heavy with respect to the lipid molecules.

Two well-known phenomena are combined here to generate and detect surface pressure waves without contacting the monolayer.

(1) Small curvature ripples that are characterized by a small amplitude-to-wavelength ratio propagate along film-covered liquid-gas interfaces in at least two modes, the transverse capillary mode and the longitudinal mode. Waves with amplitudes much smaller than the wavelength are called transverse capillary waves. The surface energy is dominant with respect to the hydrostatic pressure as a restoring force after a deviation of the surface normal to the interface. ${ }^{5}$ Periodical surface density fluctuations, i.e., longitudinal waves, only exist at film-covered liquid-gas interfaces. A resonant coupling between transverse capillary and longitudinal waves occurs for a particular surface dilatational elasticity where both waves have the same propagation velocity. ${ }^{1,4,6}$

(2) The wavelength of transverse capillary waves is strongly dependent on the surface energy. Therefore, a longitudinal wave can be detected by superposing a transverse capillary wave in such a way that their wave vectors are orthogonal. ${ }^{3,7}$

(1) Lucassen, J. Trans. Faraday Soc. 1968, 548, 2221, 2230

(2) Crone, A. H. M.; Snik, A. F. M.; Poulis, J. A.; Kruger, A. J.; van den Tempel, M. J. Colloid Interface Sci. 1980, 74, 1.

(3) Miyano, K.; Abraham, B. M.; Ting, L.; Wasan, D. T. J. Colloid Interface Sci. 1983, 92, 297.

(4) Thiessen, D.; Sheludko, A. Kolloid-Z. 1967, 218, 139.

(5) Landau, L. D.; Lifschitz, E. M. In Lehrbuch der Theoretischen Physik: Hydrodynamik, 4th ed.; Academie-Verlag: Berlin, 1982; Vol. 6, p $266 \mathrm{ff}$.

(6) Langevin, D. J. Colloid Interface Sci. 1981, 80, 412.

(7) Lucassen, J.; van den Tempel, M. Chem. Eng. Sci. 1972, 27, 1283.
The inherent coupling of transverse capillary and longitudinal waves provides a tool for longitudinal wave excitation. Transverse capillary waves can be generated without contacting the monolayer by the use of electrocapillarity: a sinusoidal voltage is applied between the water and a thin metal blade in the air close to the surface. ${ }^{8}$ The transverse capillary wave propagation is detected via specular reflection of a laser beam from the water surface.

Here, surface density fluctuations have been investigated by superposing two transverse capillary waves, $\mathrm{A}$ and $\mathrm{B}$. The wavelength of one transverse capillary wave is periodically modulated by the second wave only if the latter is inherently coupled to a longitudinal wave. ${ }^{9}$

\section{Theory}

From a complete hydrodynamic description of waves propagating along the surface of an incompressible liquid a determinant was derived ${ }^{10-13}$ with complex entities that can be solved only numerically. An approximate solution for the dispersion equation of ripples with a small amplitude-to-wavelength ratio propagating along the uncovered water surface has already been found by Kelvin.

$$
\omega_{\mathrm{T}}=\left(\sigma k_{\mathrm{T}}{ }^{3} / \rho\right)^{1 / 2}
$$

The propagation characteristics of these transverse capillary waves strongly depend on the surface energy $\sigma$.

For film-covered surfaces there exist two independent solutions for plane wave propagation: transverse capillary and longitudinal waves. ${ }^{1}$ The appearence of longitudinal waves requires a film coverage of the incompressible subphase since they originate from surface density fluctuations. The surface dilatational elasticity $\epsilon$ that measures the surface resistance against changes in the area is defined as

$$
\epsilon=A(\mathrm{~d} \sigma / \mathrm{d} A)_{\mathrm{T}}
$$

and can be directly calculated from the slope of the surface pressure/area isotherm where the surface pressure is de-

(8) Sohl, C. H.; Miyano, K.; Ketterson, J. B. Rev. Sci. Instrum. 1978, $49,1464$.

(9) Vogel, V., Dissertation, Fachbereich Physik, Frankfurt am Main 1987.

(10) Hansen, R. S.; Mann, J. A. J. Appl. Phys. 1964, 35, 152.

(11) van den Tempel, M.; van de Riet, R. P. J. Chem. Phys. 1965, 42 2769.

(12) Lucassen, J.; Hansen, R. S. J. Colloid Interface Sci. 1966, $22,32$.

(13) Lucassen-Reynders, E. H.; Lucassen, J. Adv. Colloid Interface Sci. 1969, 2, 347. 
fined as $\pi=\sigma_{\text {water }}-\sigma_{\text {monolayer }}$ Expressions for the damping constant were derived by Stokes and Reynolds for zero and infinitely high surface dilatational elasticities. At intermediate values the damping constant does not increase monotonically but passes through a maximum at low surface dilatational elasticities. Theoretical ${ }^{1,10,11}$ as well as experimental evidence is given for a large number of surfactant monolayers including lipids and polymers. ${ }^{4,6,10-16}$

An approximate dispersion relation for longitudinal wave propagation has been derived by Lucassen ${ }^{1}$

$$
\omega_{\mathrm{L}}=\left[\left(\frac{1}{2}+2^{-3 / 2}\right)^{-2} \frac{\epsilon^{2} k_{\mathrm{L}}{ }^{4}}{\eta_{\mathrm{s}} \rho}\right]^{1 / 3}
$$

The surface dilatational elasticity $\epsilon$ strongly affects the wave number of longitudinal waves whereas its influence on the dispersion of transverse capillary waves at filmcovered interfaces is relatively small.

For monolayer-covered surfaces there exists an inherent coupling between longitudinal and transverse capillary waves. A resonant coupling occurs if both excited waves have the same propagation velocity giving rise to a maximum in the damping constant of transverse waves. Since the dispersion relations of transverse capillary and longitudinal waves are affected differently by the surface dilatational elasticity, the resonance at film-covered surfaces will be found for a particular value of the surface elasticity: the reduced elasticity $\epsilon / \sigma$. It is just the ratio of the surface dilatational elasticity to the surface tension derived from the resonance condition $\left(k=k_{\mathrm{L}}=k_{\mathrm{T}}\right.$ for the same excitation frequency) ${ }^{1}$

$$
\epsilon / \sigma=\left(\frac{1}{2}+2^{-3 / 2}\right)\left(\frac{\eta_{\mathrm{s}}{ }^{2} k}{\rho \sigma}\right)^{1 / 4}
$$

\section{Experimental Section}

Generation and Detection of Transverse Capillary Waves. Electrocapillary deflection of the water/air interface is used to generate transverse capillary waves ${ }^{8}$ in a frequency range of 50-1000 $\mathrm{Hz}$. A sinusoidal voltage of about $100 \mathrm{~V}$ is applied between a thin metal blade $(70 \mathrm{~mm}$ long and $0.02 \mathrm{~mm}$ thick) and the water with the blade held at less than $1 \mathrm{~mm}$ from the surface (Figure 1a). Capillary waves excited by electrocapillarity have wavelengths in the order of $1 \mathrm{~mm}$ with up to $100 \mathrm{~nm}$ in amplitude. The deflection of the surface is detected after specular reflection of a focused $\mathrm{He} / \mathrm{Ne}$ laser beam from the water surface by a differential photodiode with linear response for small deflections (Figure 1b). The water surface is scanned over $25 \mathrm{~mm}$ perpendicular to the wave generator starting $5 \mathrm{~mm}$ apart from the blade. The distance between the metal blade and the water surface critically determines the initial amplitude of the transverse capillary waves. The exponentially decaying amplitude is recorded as function of the scanning distance (Figure 2) by the use of a lock-in technique. The slope of the logarithmic plot of the extreme values of the damped oscillations provides the damping constant $\alpha$.

At the water/air interface, the deviations between experiment and theory are less than $3 \%$ for the wavelength (eq 1) and less than $10 \%$ for the damping constant $\left(\alpha_{\mathrm{H}_{2} 0}=4 \eta_{8} \omega / 3 \sigma\right)$, in good agreement with other ripple techniques. ${ }^{19,18}$

In case of two superposed transverse plane waves, $A$ and $B$, the amplitude of wave $B$ propagating perpendicularly to the detection line was determined by rotating the position sensitive photodiode around the optical axis $\left(90^{\circ}\right)$. To simplify the generation and detection techniques, both waves $\mathrm{A}$ and $\mathrm{B}$ have been excited with the same frequency.

(14) Garnet, W. D.; Zisman, W. A. J. Phys. Chem. 1970, 74, 1797.

(15) Hansen, R. S.; Ahmad, J. Prog. Surf. Membr. Sci. 1971, 4, 1.

(16) Mann, J. A. Surf. Colloid Sci. 1984, 13, 145.

(17) Stone, J. A.; Rice, W. J. J. Colloid Interface Sci. 1977, 61, 160.

(18) Byrne, D.; Earnshaw, J. C. J. Colloid Interface Sci. 1980, 74, 467. a
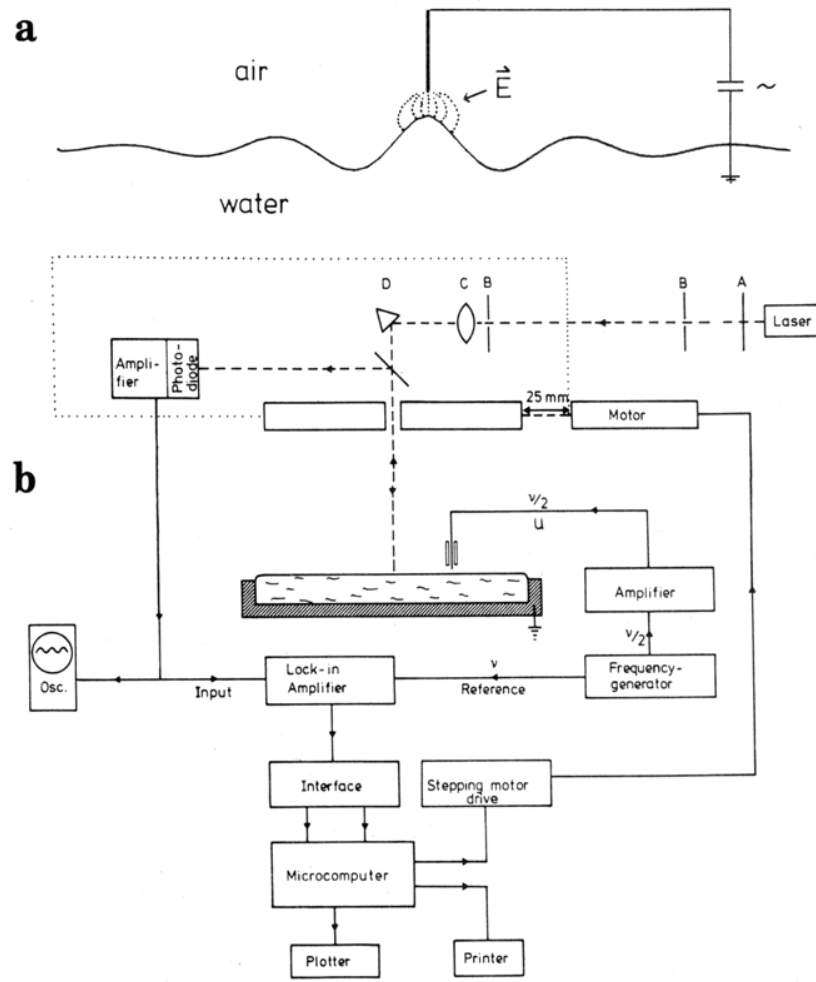

Figure 1. Generation and detection of transverse capillary waves. Transverse capillary waves are excited by an electrocapillary deflection of the interface within an inhomogeneous $\vec{E}$-field between a metal blade and the water surface (a, top part). The waves are detected by specular reflection of a laser beam from the water surface to a photodiode. The signal is amplified by using a lock-in technique and further processed by a microcomputer.

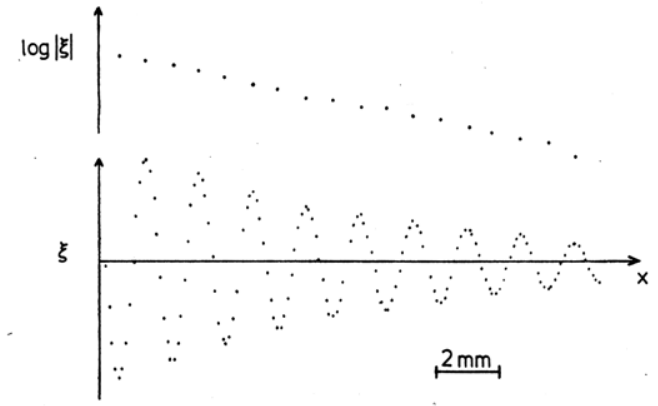

Figure 2. Damping of transverse capillary waves at the monolayer-covered water surface. The unscaled amplitude $\zeta$ and $\log$ $|\zeta|$ of the exponentially decaying wave is recorded as a function of the scanning distance $x$ perpendicular to the wave generator (film of poly(vinyl acetate), $\pi=1.2 \mathrm{mN} / \mathrm{m}, A=43 \AA^{2} /$ monomer subunit, $\nu=160 \mathrm{~Hz}, 20^{\circ} \mathrm{C}$ ).

Monolayer Preparation. A rectangular trough of poly(tetrafluoroethylene) with a depth of $10 \mathrm{~mm}$ and a surface area of $18 \times 55 \mathrm{~cm}^{2}$ enclosed in a tight box was used. Deionized water filtered by a Milli-Q System was used as subphase without the addition of any ions. The ripple characteristics of the monolayer-free water surface were determined. After spreading poly(vinyl acetate) ${ }^{19}\left(\mathrm{PVAc},-\left(\mathrm{CH}_{2} \mathrm{CH}\left(\mathrm{OCOOCH}_{3}\right)\right)_{n}^{-}\right.$, dissolved in $\mathrm{CH}_{2} \mathrm{Cl}_{2}(5 \mathrm{mg} / 10 \mathrm{~mL})$ ), we equilibrated the film for $5 \mathrm{~min}$ before stepwise compression. The surface pressure was measured by a filter paper Wilhelmy balance. The monolayer trough and the optical system were mounted on a vibrationally damped table.

\section{Detection of Resonantly Excited Longitudinal Waves}

For transverse capillary waves, the wavelength $\lambda$, the relative damping constant $\alpha_{\mathrm{M}} / \alpha_{\mathrm{H}_{2} \mathrm{O}}$, and the surface

(19) Sauer, B. B.; Kawaguchi, M.; Yu, H. Macromolecules 1987, 20, 


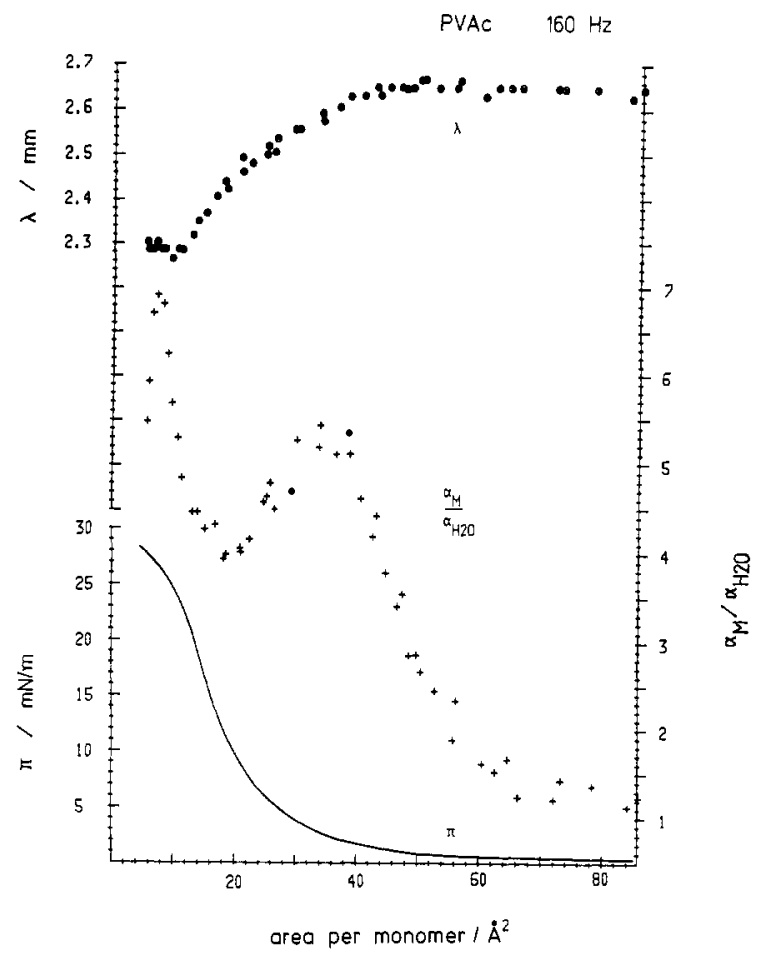

Figure 3. Propagation characteristics of transverse capillary waves at the monolayer-covered water surface. The wavelength $\lambda$, the relative damping constant of the monolayer with respect to the uncovered water surface $\alpha_{\mathrm{M}} / \alpha_{\mathrm{H}_{2} \mathrm{O}}$, and the surface pressure $\pi$ are plotted vs the mean area per monomer subunit of the polymer PVAc $\left(\nu=160 \mathrm{~Hz}, \alpha_{\mathrm{H}_{2} \mathrm{O}}=0.0163 \mathrm{~mm}^{-1}, 19^{\circ} \mathrm{C}, \mathrm{pH} 5.6\right.$, water).

pressure $\pi$ are given in Figure 3 as functions of the mean area per monomer subunit for a poly(vinyl acetate) monolayer (PVAc). The damping constant does not increase monotonically with rising surface pressures but rather passes through two maxima, one at a mean area of about $36 \AA^{2}$ /monomer subunit and the second at $9 \AA^{2}$ / monomer subunit. No indication of a phase transition is given by the surface pressure/area isotherm. From a plot of the damping constant and the reduced elasticity $\epsilon / \sigma$ (calculated from the surface pressure area isotherm, eq 2) vs the mean area per monomer subunit (Figure 4), it is obvious that the maximum in the damping is correlated to the elastic properties of the monolayer-covered water surface: the curvature of the $\pi / A$ isotherm changes gradually from positive to negative values on monolayer compression. The reduced elasticity therefore runs through two maxima for a rather small reduced elasticity. Its value of $\epsilon / \sigma=0.084$ (eq 4) given by the resonance condition correlates very well with the reduced static elasticity of $\epsilon / \sigma=0.08$ (eq 2) determined from the surface pressure/area isotherm.

To study the coupling of transverse capillary to longitudinal waves, a second wave generator is mounted orthogonally to the first (Figure 5). Two transverse capillary waves, $A$ and $B$, superpose perpendicularly to detect surface pressure fluctuation. Since the wavelength of transverse capillary waves varies with the surface pressure, the wavelength of the transversal mode of $A$ is modulated by the longitudinal mode of wave $B$ at resonance.

The low surface pressure region at areas near to 36 $\AA^{2} /$ monomer subunit was selected for the detection of longitudinal waves (see Figure 4). For small surface dilatational elasticities, longitudinal waves are more strongly damped than transverse capillary waves, but the opposite is true for large surface dilatational elasticities normally found within close-packed monolayers. ${ }^{1,3}$ Therefore, it was

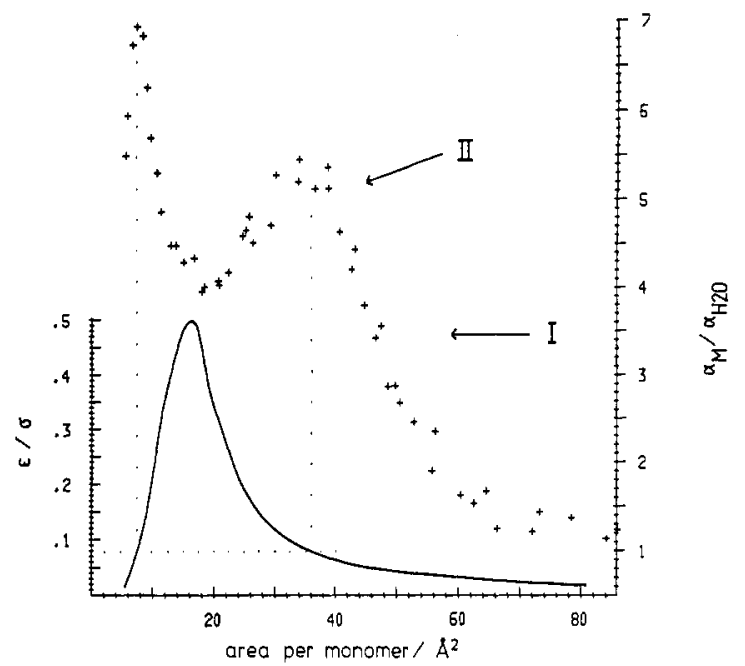

Figure 4. Two maxima occur in the damping of transverse capillary waves for a particular value of the reduced elasticity $\epsilon / \sigma$. This coupling provides a tool to excite periodical surface density fluctuations in monolayers at liquid-gas interfaces in the frequency range from $100 \mathrm{~Hz}$ to $1 \mathrm{kHz}$. The static reduced elasticity is calculated from the data given in Figure 3 (eq 2).

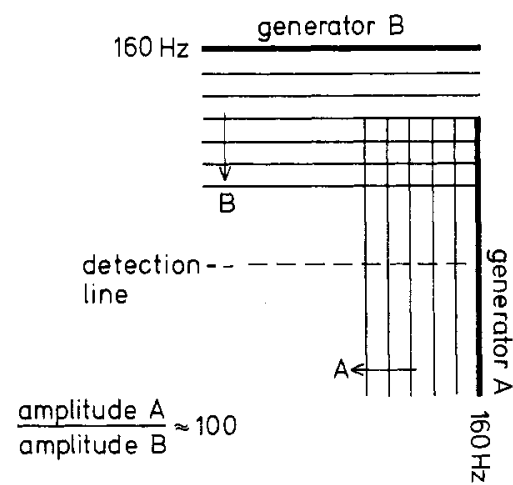

Figure 5. Detection of surface pressure fluctuations by an orthogonal superposition of two transverse capillary waves under resonance. The longitudinal mode of wave $B$ causes periodical changes of the wavelength of the transverse capillary wave $A$ via surface pressure fluctuations. With these methods a physical contact with the surface is avoided during the generation and the detection of longitudinal waves.

more interesting to prove whether longitudinal waves excited under resonance at small surface pressures are detectable by the technique presented in this paper.

It was ascertained that the superposition of wave $B$ does not influence the propagation characteristics of wave $A$ at the monolayer-free and at the film-covered surface far from resonance. The transverse amplitude of $B$ along the detection line was about $1 / 100$ th the amplitude of $\mathrm{A}$.

Indeed, modulations of the wavelength of $A$ are observed close to the damping maximum at $36 \AA^{2} /$ monomer subunit (Figure 6). The wavelength of A reacts sensitively on the surface pressure fluctuations that accompany the propagation of wave $B$. This wavelength of $A$ is plotted against the phase of wave $B$ at the detection line since a lcck-in technique is used.

It is to be expected that inherently coupled transverse capillary and longitudinal waves are phase shifted, ${ }^{4}$ as can be observed in Figure 6 . The transverse amplitude of $B$ was recorded at the detection line for different phases between wave generation and detection without applying a voltage to the wave generator A (Figure 6, upper part, $\left.\alpha_{\mathrm{m}} / \alpha_{\mathrm{H}_{2} \mathrm{O}}=3.7\right)$. Within the same plane wave, the longitudinal mode is phase shifted by about $90^{\circ}$ with respect to the transverse mode. An increase of the transverse 

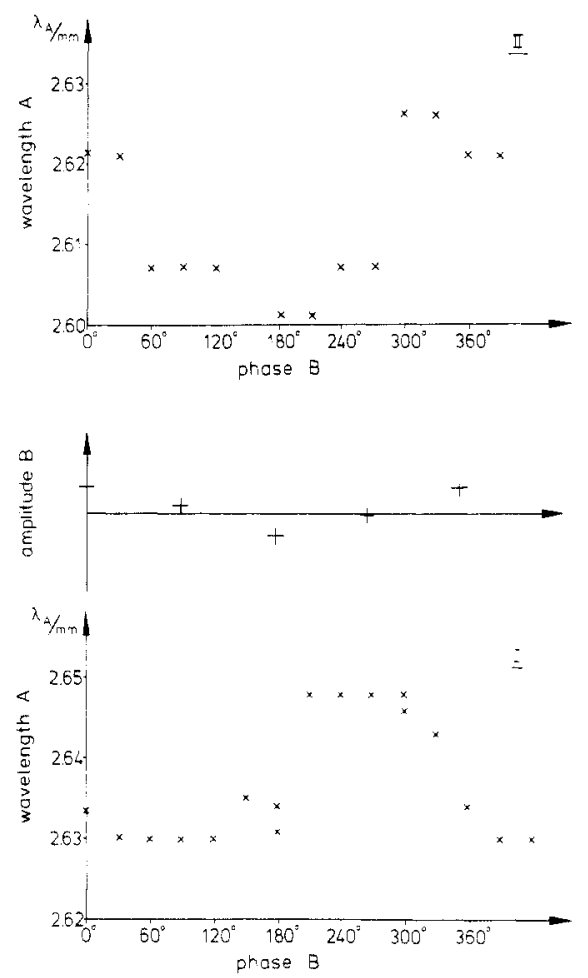

Figure 6. Resonances between transverse capillary and longitudinal waves. Near the damping maximum the wavelength of the transverse capillary wave A sensitively depends on the phase of wave $B$ crossing the detection line perpendicularly. The amplitude of the wavelength modulation increases with increasing damping (I, $\alpha_{\mathrm{M}} / \alpha_{\mathrm{H}_{2} \mathrm{O}}=3.7 ;$ II, $\alpha_{\mathrm{M}} / \alpha_{\mathrm{H}_{2} \mathrm{O}}=5$, compare with arrows in Figure 4). Further, a phase shift of about $90^{\circ}$ is observed between the wavelength of the transverse capillary wave $A$, i.e., the surface pressure maximum (lower part), and the transverse amplitude of wave $B$ (upper part). The longitudinal mode is phase shifted with respect to the transverse mode.

Table I. Perpendicular Superposition of Two Waves, $A$ and B, To Detect Periodical Surface Pressure Modulations ${ }^{a}$

\begin{tabular}{ccccc}
\hline & $\begin{array}{c}\lambda_{\min } \\
\left(\zeta_{\mathrm{B}} \neq 0\right), \\
\mathrm{mm}\end{array}$ & $\begin{array}{c}\lambda_{\mathrm{A}} \\
\left(\zeta_{\mathrm{B}}=0\right), \\
\mathrm{mm}\end{array}$ & $\begin{array}{c}\lambda_{\max } \\
\left(\zeta_{\mathrm{B}} \neq 0\right), \\
\mathrm{mm}\end{array}$ & $\begin{array}{c}\Delta \lambda, \\
\mathrm{mm}\end{array}$ \\
\hline$\alpha_{\mathbf{M}} / \alpha_{\mathrm{H}_{2} \mathrm{O}}$ & $\mathrm{mm}$ & 2.645 & 2.645 & 0.018 \\
3.7 & 2.630 & 2.621 & 2.626 & 0.025
\end{tabular}

a Phase-dependent modulation of the wavelength $\lambda_{A}$ of wave A by the longitudinal mode of wave $B$. Notice that the difference $\lambda_{A}$ $-\lambda_{\min }$ is larger than $\lambda_{\max }-\lambda_{\mathrm{A}} . \quad \lambda_{\mathrm{A}}$ is the wavelength of wave $\hat{A}$ without wave $B$ being superposed $\left(\zeta_{B}=0\right)$.

amplitude of $B$ causes an increase in surface pressure and a decrease in surface energy (eq 1 ).

The coupling between transverse and longitudinal modes is strongly enhanced close to the resonance, i.e., the damping of transverse capillary waves reaches its maximum (Figure 4). The damping constant runs through a maximum at a static surface pressure of $1.8 \mathrm{mN} / \mathrm{m}$. The surface pressure fluctuations accompanying wave $B$ give rise to a peak to peak change in the wavelength $\lambda_{\mathrm{A}}$ of $\Delta \lambda$ $=0.018 \mathrm{~mm}\left(\alpha_{\mathrm{m}} / \alpha_{\mathrm{H}_{2} \mathrm{O}}=3.7\right)$ and of $\Delta \lambda=0.025 \mathrm{~mm}$ $\left(\alpha_{\mathrm{M}} / \alpha_{\mathrm{H}_{2} \mathrm{O}}=5\right)$ close to the damping maximum (Figure 4, parts I and II, respectively). The fact that $\lambda_{B=0} \neq 1 /{ }_{2}\left(\gamma_{\min }\right.$ $\left.+\lambda_{\max }\right)$ indicates that eq 1 cannot be used to calculate the magnitude of the surface pressure fluctuations (Table I). To yield an estimate, the wavelength $\lambda_{A}$ is plotted vs the surface pressure without wave $B$ being superposed (Figure 7). Under the chosen conditions an upper limit of the longitudinal amplitude of wave $B$ is $\Delta \pi \leq 0.3 \pm 0.1 \mathrm{mN} / \mathrm{m}$ close to the damping maximum. The electrocapillary wave excitation provides the possibility to enhance the longi-

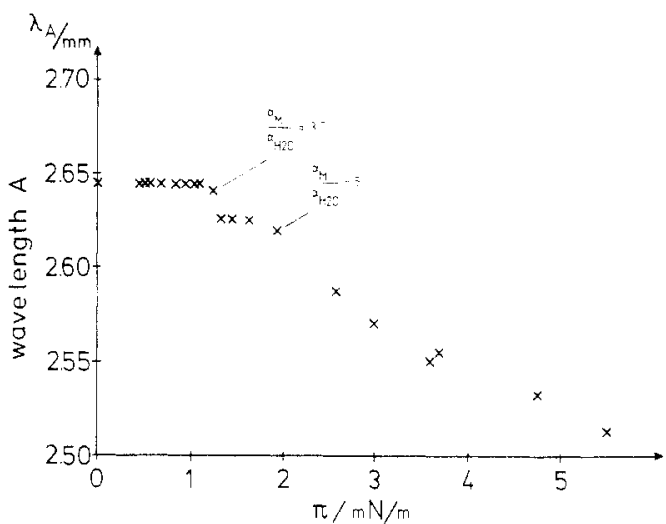

Figure 7. Dependence of the wavelength of the transverse capillary waves on the surface pressure near the damping maximum at $36 \AA^{2} /$ monomer subunit (data from Figure 3).

tudinal amplitude just by increasing externally the transverse deflection of the surface.

\section{Conclusion}

Whereas the frequency range of mechanically excited longitudinal waves is limited to low frequencies due to the inertia of the oscillators $(\nu<100 \mathrm{~Hz})$, inherent coupling of transverse capillary and longitudinal waves provides a tool for the excitation of periodical surface pressure fluctuations near the resonance at much higher frequencies. The frequency range between $100 \mathrm{~Hz}$ and $1 \mathrm{kHz}$ is excessible by electrocapillary deflection of the surface. Surface roughening by thermal fluctuations also induces transverse capillary waves but with even higher frequencies $(1 \mathrm{kHz}<\nu<50 \mathrm{kHz}){ }^{6,16-20}$

The generation of single frequent longitudinal waves by using resonant coupling of transverse capillary and longitudinal waves is complementary to the generation of aperiodical surface pressure pulses by photoisomerization of molecules embedded into lipid monolayers, ${ }^{21,22}$ e.g., a lnng chain substituted spiropyran. This has a smaller area pe ${ }_{1}$ molecule than the photoisomerization product merocyanine. Under constant surface area, the light-induced molecular reorganization generates a surface pressure jump. The frequency spectrum of such two-dimensional surface pressure pulses has not yet been determined since the contact-free detection in the case of single surface pressure pulses was not possible.

It has been shown here that periodical surface pressure fluctuations can be investigated for polymer or lipid monolayers at the air/water interface by the orthogonal superposition of two transverse capillary waves. Relaxation processes within monolayers as well as surface pressure dependent partitioning of molecules at the interface between lipids and the aqueous subphase are of great biological relevance in the frequency range that is accessible by electrocapillary wave excitation. For example, usual time constants for the opening of membrane channels are within milliseconds. Molecules or molecular aggregates of special interest can be embedded in a suitable monolayer matrix.

Acknowledgment. We thank Prof. J. C. Earnshaw and Prof. J. A. Mann for fruitful discussions, H. Meyer for his help in the construction of the apparatus, and Prof. $\mathrm{H}$. Yu for supplying us with the polymer.

20) Hard, S. Neuman, R. D. J Colloid Interface Sci. 1987, 115, 73 (21) Möbius, D.; Grüniger, H. R. In Charge and Field Effects in Biosystems; Allen, M. J., Usherwood, P. N. R., Eds.; Abacus: Tunbridge Wells, England, 1984; p 265. 151.

(22) Suzuki, M.; Möbius, D.; Ahuja, R. Thin Solid Films 1986, 138, 


\begin{tabular}{llll} 
& \multicolumn{1}{c}{$\begin{array}{c}\text { Symbols } \\
A\end{array}$} & $\zeta$ & amplitude of the transverse mode \\
$\alpha$ & area per molecule $\left(\AA^{2}\right)$ & $\pi$ & surface pressure $(\mathrm{mN} / \mathrm{m})$ \\
$\lambda$ & damping constant $\left(\mathrm{mm}^{-1}\right)$ & $\rho$ & density of the solution $\left(\mathrm{kg} \mathrm{m} \mathrm{m}^{-3}\right)$ \\
$\lambda$ & wavelength $(\mathrm{mm})$ & $\sigma$ & absolute surface tension $(\mathrm{mN} / \mathrm{m})$ \\
$\epsilon$ & surface dilatational elasticity $(\mathrm{mN} / \mathrm{m})$ & $T$ & temperature \\
$k$ & wave number $\left(\mathrm{mm}^{-1}\right)$ & $\omega$ & angular frequency $\left(\mathrm{s}^{-1}\right)$ \\
$\eta_{\mathrm{B}}$ & viscosity of the solution $(\mathrm{mP})$ & \multicolumn{2}{c}{ Registry No. PVA, 9003-20-7. }
\end{tabular}

\title{
Dynamic Behavior of Single Glycerol Droplets in Humid Air Streams
}

\author{
A. K. Ray,* R. D. Johnson, and A. Souyri \\ Department of Chemical Engineering, University of Kentucky, \\ Lexington, Kentucky 40506-0046 \\ Received January 26, 1988. In Final Form: August 12, 1988
}

\begin{abstract}
The growth and evaporation of single glycerol droplets have been examined at various relative humidities. The experiments were conducted in an electrodynamic balance where a charged droplet was suspended in the path of a He-Ne laser beam in a stream of air with precisely controlled humidity and temperature. The droplet size and mass as functions of time have been obtained from two independent methods based on angular and fixed angle light-scattering data and balancing dc voltage data. The intensity (i.e., resonant) peaks observed in the experimental scattering data obtained at a fixed angle have been interpreted by using Lorenz-Mie theory to obtain droplet size and refractive index (i.e., composition) with high precision. The growth and evaporation kinetics of the same droplet have been obtained at various humidity levels by altering the humidity level of the air stream in steps. After each step increase in humidity, the droplet grew to a maximum size and then evaporated. The experimental data indicate the following: (i) The time required by a droplet to reach the maximum size is considerably higher than the time predicted by diffusion theory. (ii) During the evaporation period, for a given humidity, the composition of the droplet remains constant, and the square of the droplet radius changes linearly with time. (iii) The maximum water content in a droplet is always lower than the bulk equilibrium value and depends on the size of the droplet.
\end{abstract}

\section{Introduction}

The interaction of hygroscopic droplets with water vapor is an important phenomenon in many atmospheric and industrial processes and in aerosol particles entering the respiratory system. A critical examination of the processes that control the transfer of water molecules to and from the droplets is essential to the understanding of the growth and evaporation kinetics of multicomponent aerosols. Although a considerable amount of theoretical and experimental studies has been devoted to the evaporation and growth of single-component droplets (see ref 1-4 for reviews), only a few experimental studies have considered the evaporation and growth of droplets having more than one component. Most of the studies on multicomponent systems have been conducted either on nonvolatile solutes growing in a humid environment or on binary droplets evaporating in a vapor-free atmosphere. In the former situation the transport is limited to one species (i.e., water molecules), and in the latter case the transport takes place only from the droplet phase to the gas phase. In the following section we will briefly review the recent studies on multicomponent droplets.

Several studies ${ }^{5-11}$ have been conducted on the interaction of water vapor with solution droplets of various salts and nonvolatile solutes. Some of these studies ${ }^{5-8}$ have been devoted entirely to the understanding of the thermody. namics and phase transformations of salt-water systems. In a similar study, Rubel $^{9}$ has measured the equilibrium

\footnotetext{
*Author to whom correspondence should be sent.
}

water content in phosphoric acid droplets as a function of relative humidity. Richardson et al. ${ }^{10}$ and Sageev et al. ${ }^{11}$ have studied the growth and evaporation of sodium chloride and ammonium sulfate solution droplets. They have used gas-phase heat- and mass-transfer models to analyze the transient response of the droplets to periodic heating. In these studies, the droplet phase is assumed to be well mixed during the transient evaporation and growth periods. Growth studies on sulfuric acid droplets raise questions about the droplet phase mixing time and about the applicability of the bulk-phase equilibrium data to submicron droplets. The data of Coutarel et al. ${ }^{12}$ indicate that the growth of submicron sulfuric acid droplets when exposed to water vapor does not reach completion even after

(1) Fuchs, N. A. Evaporation and Droplet Growth in Gaseous Media; Pergamon: New York, 1959.

(2) Mason, B. J. The Physics of Clouds; Clarendon: Oxford, 1971

(3) Ray, A. K., M.S. Thesis, Clarkson College of Technology, Potsdam, NY, 1977.

(4) Davis, E. J.; Ravindran, P.; Ray, A. K. Chem. Eng. Commun. 1980, $5,251$.

(5) Richardson, C. B.; Kurtz, C. A. J. Am. Chem. Soc. 1984, 106, 6615

(6) Richardson, C. B.; Spann, J. F. J. Aerosol Sci. 1984, 15, 563.

(7) Spann, J. F.; Richardson, C. B. Atmos. Environ. 1985, 19, 819. 430.

(9) Rubel, G. O. J. Aerosol Sci. 1981, 12, 551

(10) Richardson, C. B.; Lin, H. B.; McGraw, R.; Tang, I. N. Aerosol Sci. Technol. 1986, 5, 103.

(11) Sageev, G.; Flagan, R. C.; Seinfeld, J. H.; Arnold, S. J. Colloid Interface Sci. 1987, 113, 421.

(12) Coutarel, L.; Matijevic, E.; Kerker, M.; Huang, C.-M. J. Colloid Interface Sci. 1967, 24, 338 .

(C) 1989 American Chemical Society 